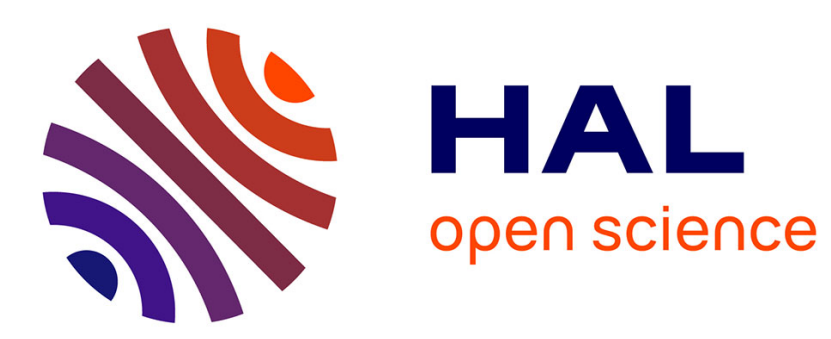

\title{
Les effets d'un instrument d'écriture à l'épreuve de la recherche. Deux ou trois choses que l'on sait ou que l'on ne sait pas sur le traitement de texte
}

Sylvie Plane

\section{- To cite this version:}

Sylvie Plane. Les effets d'un instrument d'écriture à l'épreuve de la recherche. Deux ou trois choses que l'on sait ou que l'on ne sait pas sur le traitement de texte. Repères, 2002, 26 (1), pp.163-186. 10.3406/reper.2002.2399 . halshs-03131820

\section{HAL Id: halshs-03131820 \\ https://shs.hal.science/halshs-03131820}

Submitted on 4 Feb 2021

HAL is a multi-disciplinary open access archive for the deposit and dissemination of scientific research documents, whether they are published or not. The documents may come from teaching and research institutions in France or abroad, or from public or private research centers.
L'archive ouverte pluridisciplinaire HAL, est destinée au dépôt et à la diffusion de documents scientifiques de niveau recherche, publiés ou non, émanant des établissements d'enseignement et de recherche français ou étrangers, des laboratoires publics ou privés. 


\section{Les effets d'un instrument d'écriture à l'épreuve de la recherche.} Deux ou trois choses que l'on sait ou que l'on ne sait pas sur le traitement de texte

Sylvie Plane

\section{Citer ce document / Cite this document :}

Plane Sylvie. Les effets d'un instrument d'écriture à l'épreuve de la recherche. Deux ou trois choses que l'on sait ou que l'on ne sait pas sur le traitement de texte. In: Repères, recherches en didactique du français langue maternelle, $\mathrm{n}^{\circ} 26-27,2002$. L'écriture et son apprentissage à l'école élémentaire. pp. 163-186;

doi : https://doi.org/10.3406/reper.2002.2399

https://www.persee.fr/doc/reper_1157-1330_2002_num_26_1_2399

Fichier pdf généré le 04/05/2018 


\title{
Résumé
}

Cette contribution prend comme point de départ les conclusions des historiens de la culture et des ergonomes qui ont montré que les instruments d'écriture jouaient un rôle dans l'évolution des pratiques d'écriture. Elle pose donc comme hypothèse que le traitement de texte, en tant qu'outil graphique et scriptural, est un instrument susceptible d'exercer une influence sur l'écriture et examine les résultats dégagés des recherches en fonction de leur degré de certitude. Les résultas les mieux établis concernent le rôle positif exercé par le traitement de texte sur la motivation des scripteurs. En revanche, l'effet du traitement de texte sur les processus rédactionnels n'a pas pu être mis en évidence de façon incontestable, pour des raisons qui tiennent, d'une part, à différents types de cause qui sont analysées dans cet article, mais aussi, d'autre part, aux conceptions de l'écriture qui ont présidé aux expérimentations. Enfin, l'article recense cinq points sur lesquels il appelle le développement de recherches qui éclaireraient la connaissance lacunaire que l'on a actuellement de l'écriture sur traitement de texte.

\begin{abstract}
The effects of a writing instrument put to the test of research. Two or three things known or unknown on word processor.

Sylvie Plane, LEAPLE (UMR 8086 CNRS -Université Paris V) \& INRP, IUFM de Paris.

This contribution takes as starting point the conclusions of culture historians and of ergonomists who showed that writing instruments played a role in the evolution of writing practices. It sets as hypothesis that the word processor, as a graphie and scriptural tool, is a tool likely to influence the writing and examine the results brought out by the researches according to their degree of certainty. The bestestablished results concern the positive role exercised by the word processor over the motivation of the writer. On the other hand, the effect of word processor on the editorial processes could not be brought to the fore in an unquestionable way, for reasons, which on one hand are due to different types of causes that are analysed in this article, but also on the other hand due to the conceptions of writing that have governed the experimentations. Finally, the article lists five points according to which it appeals for the development of researches, which would enlighten the lacunary knowledge we have actually on writing with word processors.
\end{abstract}




\title{
LES EFFETS D'UN INSTRUMENT D'ÉCRITURE À L'ÉPREUVE DE LA RECHERCHE Deux ou trois choses que l'on sait ou que l'on ne sait pas sur le traitement de texte
}

\author{
Sylvie PLANE - IUFM de Paris - LEAPLE (CNRS-Université Paris V) \\ Chercheur associé à l'INRP
}

\begin{abstract}
Résumé : Cette contribution prend comme point de départ les conclusions des historiens de la culture et des ergonomes qui ont montré que les instruments d'écriture jouaient un rôle dans l'évolution des pratiques d'écriture. Elle pose donc comme hypothèse que le traitement de texte, en tant qu'outil graphique et scriptural, est un instrument susceptible d'exercer une influence sur l'écriture et examine les résultats dégagés des recherches en fonction de leur degré de certitude. Les résultas les mieux établis concernent le rôle positif exercé par le traitement de texte sur la motivation des scripteurs. En revanche, l'effet du traitement de texte sur les processus rédactionnels n'a pas pu être mis en évidence de façon incontestable, pour des raisons qui tiennent, d'une part, à différents types de cause qui sont analysées dans cet article, mais aussi, d'autre part, aux conceptions de l'écriture qui ont présidé aux expérimentations. Enfin, l'article recense cinq points sur lesquels il appelle le développement de recherches qui éclaireraient la connaissance lacunaire que l'on a actuellement de l'écriture sur traitement de texte.
\end{abstract}

\%

Le traitement de texte est un instrument d'écriture qui a pour particularité d'être à la fois un outil graphique - il prolonge le geste physique en traçant une forme - et un outil scriptural puisqu'il facilite les opérations de transformation textuelle et la diffusion de l'écrit. À ce double titre, il est probable qu'il jouera un rôle déterminant dans l'évolution des multiples dimensions de l'écriture qui font d'elle, selon les niveaux où on la regarde, une technique de communication inscrite dans un système culturel, une pratique sociale, une activité cognitivo-langagière, ou un système sémiotique.

En effet, les historiens de la culture et les anthropologues ont montré comment, à différentes reprises, les changements d'outils graphiques ont influé sur les systèmes et les pratiques d'écriture. Cette question a d'abord été explorée d'un point de vue historique ou ethno-culturel, dans le cadre de travaux s'intéressant à l'évolution des modes de symbolisation et aux liens entre les fonctionnements sémiotiques induits par les techniques d'écriture, les types de formalisation qu'elles autorisent et les univers culturels des différentes civilisations. Ces études 
avaient alors pour visée soit d'établir la genèse des sysèmes sémiotiques (entreprise de démonstration par Gelb (1963/1973) de la monogenèse de l'écriture phonétique), soit de repérer les traces des contacts culturels entre populations (par exemple, controverse entre Février, Cohen et Filiozat sur l'origine des variations graphiques, en particulier les ligatures, ou dispositionnelles qui ont affecté les écritures issues de l'Araméen lors de leur adoption par les Palmyréniens et les Nabatéens (Cohen, 1963)), soit d'analyser les rapports entre des formes culturelles et des modes de pensée (Goody 1977/1979; Olson, 1994/1998).

Plus récemment, la question du rôle de l'instrument a été envisagée sous l'angle de son traitement cognitif. En effet, si on se réfère à la définition que Rabardel (1997) donne de l'instrument, voyant en lui une entité bi-face composée d'un artefact matériel ou symbolique et des schèmes d'utilisation qui lui sont associés, on est amené à considérer que l'apparition d'un nouvel outil a des conséquences d'ordre cognitif ${ }^{1}$. La psycho-ergonomie rejoint ici les conclusions de l'anthropologie, et en particulier celles de Leroi-Gourhan (1964) qui s'est attaché, dans une perspective paléontologique, à mettre en évidence les fonctions de l'outil dans le développement humain en dénonçant les présupposés idéologiques sur lesquels repose l'idée communément admise de la priorité du développement du cerveau sur celle du dispositif corporel que celui-ci contrôle. Les nouveaux media informatiques ont donc été accueillis comme des instruments dont l'usage influe sur l'utilisateur, et se sont trouvés à la source de travaux portant sur la manière dont est traitée l'information à partir de documents hypertextuels ou hypermédias (Lévy, 1990a et b ; Laufer et Scavetta, 1992 ; Rouet, 1997, 2000 ; Anis, 1998 ; Crinon et Pachet, 1995 ; Crinon et Legros, 2001 ; Mangenot, 1995, 1996, 2001 ; Godinet, 2000). La notion d'outil a été également été revisitée par des travaux inscrits dans le champ de la psychologie du développement à l'occasion de recherches s'intéressant à la genèse des processus sémiotiques, la réflexion se centrant alors autour de la définition de la notion d'outil sémiotique, examiné dans une perspective conjuguant des approches peircéennes et des approches vygostskiennes (Moro, Schneuwly et Brossard 1997 ; Schneuwly et Pfeiffer, 2001).

Je partirai donc de l'hypothèse que l'utilisation du traitement de texte peut exercer une influence sur l'écriture - et partant sur son apprentissage - et j'explorerai cette hypothèse en déclinant les questions qu'elle a suscitées et surtout celles qui se posent aujourd'hui, et en les regroupant non par thème, mais par niveau d'incertitude.

\section{SUR QUELS POINTS A-T-ON DES CERTITUDES OU DES QUASI-CERTITUDES?}

L'exposé des thèmes sur lesquels on dispose de certitudes concernant les effets du traitement de texte sera fort bref, puisque seuls les aspects affectifs de l'utilisation de l'informatique sont concernés.

1. Voir une analyse didactique dans Plane S. et Schneuwly B. (2001), "Regard sur les outils de l'enseignements du français - Un premier repérage ", Repères 21. 
En effet, l'unique acquis consensuel des recherches consacrées aux effets du traitement de texte porte sur les motivations des scripteurs. La plupart des recherches portant sur ce thème sont déjà anciennes, mais elles soulignent avec constance que le recours au traitement de texte exerce un pouvoir attractif chez les scripteurs, et en particulier chez les scripteurs adolescents.

Dans sa revue de littérature portant sur l'enseignement de la production écrite sur ordinateur, Borgognon (1998) signale bon nombre d'auteurs qui s'accordent sur l'idée que " d'une manière générale les étudiants ont une attitude positive lorsqu'ils utilisent le traitement de texte pour écrire ". Et depuis les premiers travaux de Papert (1981) qui saluait avec enthousiasme le fait que l'ordinateur ressuscitait une appétence à l'écriture chez des élèves en froid avec la production d'écrits, les recherches n'ont pas cessé de mettre en évidence le pouvoir de réconciliation avec l'écriture exercé par le traitement de texte auprès d'enfants (Pouder, 1995) ou d'adolescents (Bayard \& Nicolet, 1998)

La chose a été vérifiée expérimentalement : la plupart des recherches ${ }^{2}$ comportant un volet consacré à l'étude de la motivation ou des jugements de satisfaction des scripteurs écrivant sur traitement de texte attestent d'un taux de satisfaction dans les groupes expérimentaux supérieur à celui enregistré dans les groupes témoins (par exemple Lusignan et Fortier, 1990 ; test S.E.U.T de Préfontaine, 1987) ; et elle est confirmée quotidiennement par l'observation ordinaire empirique.

Les causes sont faciles à identifier. Le prestige de l'instrument informatique est toujours vivace, comme j'ai pu le constater à chacune de mes visites d'ateliers informatiques, et comme me l'ont confirmé les enseignants responsables de ces ateliers destinés à des élèves en très grande difficulté ${ }^{3}$. Plus précisément, en ce qui concerne l'écriture, deux causes de l'appréciation positive du traitement de texte sont constamment relevées : la qualité de la présentation et la facilitation de la réécriture.

La qualité de la présentation apparait comme quelque chose d'externe à l'écriture, mais il convient de dépasser ce jugement sommaire. L'apparence de l'écriture fait aussi partie de ses caractéristiques structurelles, car c'est, ne l'oublions pas, une substance graphique matérialisée qui a pour propriété d'être appréciée par la vue. On sait en outre que dans les représentations que les enfants se font de la qualité de l'écriture, la propreté et la netteté sont des caractéristiques très valorisées, sans doute sous l'influence de recommandations pédagogiques, qui peuvent d'ailleurs constituer des obstacles à la révision (Plane, 1996). Mais cette association entre propreté graphique et qualité du produit scriptural n'est pas l'apanage des seuls enfants, car, comme le montrent les enquêtes menées dans le cadre de la docimologie (depuis celles de Piéron, rap-

2. Sources : données de la banque INRP-DAF (Didactique et Acquisition du Français Langue maternelle).

3. Ateliers "Lecteurs92.net " mis en place dans des collèges du Département des Hauts de Seine et animés par des enseignants volontaires. 
portées par de Landsheere, 1974), la qualité de la présentation graphique influe sur le jugement que les adultes exercent sur les textes qui leur sont soumis.

Les facilitations techniques de réécriture ont elles aussi été depuis longtemps mis en avant, notamment par Daiute (1981), et soulignées dans toutes les enquêtes faites auprès des usagers, enfants ou adultes, qui, à l'unisson, se félicitent d'être dispensés par le traitement de texte de la fastidieuse recopie même si par ailleurs l'activité de copie peut en soi être intéressante et appréciée comme le montre Barré de Miniac (1999).

Sur ces points, le résultat des recherches ne fait donc que confirmer ce que le sens commun permettait de supposer.

\section{SUR QUELS POINTS PEUT-ON PENSER QUE L'ON N'AURA JAMAIS DE CERTITUDE?}

Il est deux autres points, liés entre eux, sur lesquels les recherches ont été nombreuses, mais n'ont pas apporté de résultats décisifs. II s'agit de l'effet mélioratif qu'aurait le traitement de texte sur les processus rédactionnels et sur la qualité du texte produit. Les deux préoccupations sont bien évidemment en étroite dépendance : on est droit de penser que toute action sur les processus rédactionnels, aura des effets en termes d'amélioration ou de détérioration sur la qualité du texte produit. La question de la " qualité " du texte a d'ailleurs été envisagée soit comme un thème de recherche à part entière, soit comme un indicateur renseignant sur l'évolution des processus rédactionnels. Sur ces thèmes, un très grand nombre d'expérimentations ont été réalisées, et les données qui en sont issues ont été examinées par Piolat puis par Crinon et Mangenot, si bien qu'on dispose actuellement de résultats analysés et aisément consultables, que j'évoquerai plus loin.

\subsection{Des hypothèses concordantes, mais qui laissent des blancs}

Deux grandes hypothèses différentes ont été formulées à propos des effets du traitement de texte sur les processus rédactionnels, référées l'une et l'autre au modèle de Hayes et Flower $(1980)^{4}$. L'hypothèse la plus fréquemment développée est celle d'une influence positive du recours au traitement de texte sur la révision ; l'autre hypothèse, formulée postérieurement à la précédente, concerne les effets du traitement de texte sur la planification (Espéret, 1991 ; Espéret et Crété, 1995). II faut également ajouter à ces hypothèses celle formulée par Snyder (1993a et b in Espéret et Crété 1995), selon laquelle le recours au traitement de texte faciliterait la planification, la composition et la révision. On note à ce sujet l'hétérodoxie de cette dernière proposition qui associe la composition à deux macro-processus censés ne pas être sur le même plan.

4. Rappelé dans l'article de Michel Fayol et Claudine Garcia-Debanc publié dans ce numéro de Repères. 
Ce rapide rappel incite à deux remarques. La première concerne le fait que, seule des trois macro-processus isolés par Hayes et Flower, la textualisation (module translating dans le modèle de Hayes et Flower) apparaît comme non sensible aux effets du traitement de texte. L'absence de la textualisation au nombre des macro-processus affectés positivement peut recevoir trois explications :

- La centration très forte sur la révision dont le fonctionnement venait d'être mis à jour a fait que l'attention des chercheurs s'est massivement portée sur ce processus au détriment des autres. En effet, la révision a été décrite comme caractéristique de l'expertise rédactionnelle (cf. revue de travaux de Fayol et Gombert, 1987), et les travaux menés dans le cadre de la psychologie expérimentale, tout comme ceux menés dans le cadre de la didactique ont mis en évidence des déficits dans ce domaine chez des scripteurs malhabiles ou débutants (Rosat, Dolz et Schneuwly, 1991 ; Turco, Plane et Mas, 1994).

- D'une façon générale, les travaux de psycholinguistique qui ont pris comme outil conceptuel de référence le modèle de Hayes et Flower se sont assez peu intéressés aux problèmes de textualisation proprement dit, du moins ils n'en ont fait pas le centre de leurs préoccupations. Fabre-cols $(1990,2002$ ) souligne d'ailleurs les faiblesses dans le domaine linguistique de certaines analyses psycholinguistiques ${ }^{5}$, faiblesses qui tiennent au fait que, comme le notent Coirier, Gaonac'h et Passerault (1996) dans leur synthèse consacrée aux approches cognitives de la production et de la compréhension, les aspects d'ordre linguistique ne constituent qu'un des quatre types de déterminations impliquées dans les processus de production et de réception, à côté des aspects relatifs à la structuration mentale du domaine de référence, au contexte de la tâche et aux structures cognitives.

- La textualisation est en soi un processus difficile à cerner. Du moins c'est un processus pour lequel le modèle de Hayes de Flower n'est pas assez puissant, même s'il a le mérite de le prendre en considération. Dans ce modèle la textualisation apparaît un peu en creux, comme ce qui ne relève ni de la planification, ni de la révision.

La deuxième remarque concerne les limites (ou même les faiblesses) de l'étayage argumentatif de ces hypothèses relatives aux effets du traitement de texte sur les processus rédactionnels, limites qui sont à mettre en rapport avec le contexte dans lequel ont été formulées ces hypothèses. En effet, elles ont été émises dans la décennie $80-90$, et au début des années 90 , c'est-à-dire à une époque où, d'une part, la formalisation des modèles de production écrite incitait à penser qu'on allait pouvoir les enseigner aisément et améliorer ainsi les capacités rédactionnelles des étudiants, et où, d'autre part, le développement de l'informatique et sa mise à disposition du grand public s'accompagnaient d'une grande confiance placée dans ces nouveaux outils. En conséquence, les arguments permettant de fonder les hypothèses en faveur d'une influence positive

5. Fabre fait en particulier une analyse critique des travaux de Shui sur l'acquisition et de la grille d'analyse de Faigley et Witte. 
du traitement de texte sur les processus rédactionnels ont été relativement peu développés, grâce au consensus général sous-tendant ces hypothèses, qui permettait alors d'écrire : " L'ordinateur, nous le savons tous facilite la révision, mais il ne l'enseigne pas[... $]^{6}$ " (je souligne), comme si, de ces deux assertions, seule celle concernant la nécessité d'un enseignement de la révision avait besoin d'être étayée, l'autre, portant sur les vertus de l'ordinateur, apparaissant comme issue d'un consensus incontestable.

Les hypothèses qui envisagent que le traitement de texte induirait des changements processuels, qu'il s'agisse de la planification ou de la révision, se fondent sur les quatre arguments suivants, qui ont été abondamment développés et, que j'ai commentés et explicités ailleurs ${ }^{7}$ :

\section{- L'ordinateur facilite des procédures}

Les traitements de texte proposent un certain nombre de fonctionnalités qui facilitent les quatre opérations textuelles majeures entrant dans les procédures de révision : l'ajout, la suppression, le remplacement, le déplacement. Alors que dans l'écriture manuscrite ces opérations exigent un travail important de recopie, le traitement de texte prévoit pour chacun de ces opérations une manipulation technique qui allège la tâche et l'accélère (touches ou combinaison de touches permettant l'insertion, l'effacement, le couper-coller etc.)

En outre, la rapidité d'exécution permet au scripteur de multiplier et de combiner ces procédures entre elles.

\section{- L'ordinateur libère des actes moteurs de l'écriture}

Cette hypothèse avancée par Daiute et abondamment reprise, postule que le traitement de texte libère des actes moteurs de l'écriture, et permet donc au scripteur d'allouer plus de ressources cognitives aux opérations de haut niveau, telles que la recherche des idées.

Ces deux types de fonctionnalités de l'ordinateur sont en fait très liées : dans les deux cas, il est attendu de l'ordinateur qu'il dispense le sujet d'une partie de la tâche d'écriture envisagée dans sa dimension gestuelle, laquelle est considérée comme entrant en concurrence avec la partie proprement scripturale de la production d'écrit. Ainsi le modèle de production convoqué est plus complexe que celui qui sert de référence affichée : alors que le modèle de Hayes et Flower ne s'intéresse qu'aux macro-processus rédactionnels et à leurs composantes, les hypothèses que je viens d'évoquer prennent en compte des opérations cognitives dites de bas niveau et des aspects de la temporalité de

6. Bisaillon, 1991. Le propos de Bisaillon n'étant pas d'étudier les effets de l'ordinateur mais de définir des stratégies d'enseignement de la révision, il n'y a rien d'étonnant à ce qu'elle considère comme acquis le contenu d'affirmations sur lesquelles existait alors un large accord.

7. Cf. PLANE S., 1994, Didactique et pratiques d'écriture - Écrire au collège Nathan et PLANE S., 1996, "Le traitement de texte pour apprendre à réécrire " in GROUPE EVA De l'évaluation à la réécriture Hachette-INRP, 157-183. 
l'écriture qui ne figuraient dans le modèle initial. Cette argumentation ne prend donc sens que dans le cadre des modèles de productions qui, comme ceux de van Galen ${ }^{8}$, envisagent l'écriture à la fois dans ses dimensions graphiques et scripturales.

\section{- L'ordinateur offre des aides}

Cet argument renvoie au fait que les traitements de texte mettent à disposition de l'utilisateur des correcteurs orthographiques ou des dictionnaires ; les premiers allégeant la charge qu'impose la vigilance orthographique, les seconds offrant l'avantage de procurer des ressources immédiatement accessibles.

Cet argument, ainsi que les deux précédents, repose sur l'hypothèse de la surcharge cognitive qu'impose l'acte d'écriture en obligeant le sujet scripteur à régler simultanément des problèmes qui entrent en concurrence. Cette hypothèse est étayée par les nombreuses expérimentations qui ont soit montré les limites de la mémoire de travail, soit les perturbations occasionnées par les contraintes de traitements simultanés, tels que le modèle de De Beaugrande (1982, 1984), par exemple, les figure.

\section{- L'ordinateur offre une réactivité stimulante}

Un autre argument qui a été moins développé que les précédents apparaît cependant comme sous-jacent à la plupart des hypothèses optimistes en faveur d'une influence positive du traitement de texte sur les processus rédactionnels, il s'agit de l'argument développé par Papert (1981) qui considérait la machine comme investie de possibilités d'interactions plus riches que celles que permet l'environnement.

\subsection{Des résultats qui ne corroborent pas les hypothèses initiales}

En référence aux hypothèses que je viens d'exposer, des recherches ont été entreprises, pour vérifier expérimentalement si le recours au traitement de texte avait une incidence positive sur les processus de révision, ou de planification. Les observables collectés qui permettaient de confirmer ou d'invalider les hypothèses de recherche étaient de trois ordres :

- Le nombre et la nature des changements opérés sur les textes en cours de scription, et le type d'unités linguistiques concernés par ces changements.

- Le nombre et la durée des pauses, en référence aux travaux de Matshuhashi (1982) qui avait présenté les pauses comme des indices renseignant sur l'activité cognitive du scripteur et signalant que celuici était en train de traiter une difficulté ; ce sont d'ailleurs ces travaux qui ont mis en évidence la complexité processuelle de l'activité de production d'écrits qui exige la gestion simultanée ou quasi simulta-

8. Cf. l'article de Fayol et Garcia-Debanc dans ce numéro de Repères. 
née d'opérations relevant de différents niveaux de traitement, ainsi qu'il a été rappelé plus haut.

- La qualité des textes obtenus.

Piolat a fait un recensement très précis de l'ensemble des recherches expérimentales visant à vérifier les hypothèses énoncées plus haut et en a collationné les résultats (Piolat, 1991a, 1991b ; Piolat et Roussey, 1991 ; Piolat, Isnard, Della Valle, 1993). Ce recensement, qui porte sur une cinquantaine de recherches effectuées entre 1980 et 1985, fait apparaitre que contrairement aux attentes, les résultats des comparaisons entre groupes expérimentaux écrivant sur traitement de texte et groupes témoins écrivant à la main ne confirmaient pas les hypothèses en faveur d'une amélioration des processus rédactionnels qui serait due à l'utilisation du traitement de texte. Les recherches menées ultérieurement, avec des méthodologies sensiblement comparables ${ }^{9}$ corroborent les résultats enregistrés par Piolat. Le recensement effectué également par Crinon et Legros (2001), auquel je me réfère plus loin, va dans le même sens.

Si l'on reprend le premier des indicateurs, à savoir les changements opérés sur les textes, on note que, globalement, les rapports de recherche font état d'un nombre plus grand de corrections opérées par les scripteurs utilisant le traitement de texte mais que ces corrections portent sur de petites unités du texte, c'est-à-dire sur des aspects locaux, et ne concernent pas ou peu la structure du texte. C'est ce qui peut se lire par exemple dans la conclusion du rapport de Préfontaine (1987) sur les effets de l'utilisation du traitement de texte sur la pratique de l'écriture par des adolescents :

Au niveau des erreurs profondes, il y a très peu de différence entre les résultats des deux groupes, les plus importantes portant sur l'introduction et la conclusion, où les élèves du groupe expérimental font de meilleures introductions, alors que les élèves du groupe contrôle font de meilleures conclusions. Toutefois ces résultats sont trop peu significatifs pour que nous puissions en tirer une signification satisfaisante.

Dans d'autres recherches qui avaient pris les temps de pause comme indicateurs, les résultats sont été également très mitigès, et n'ont guère permis d'arriver à des conclusions déterminantes (par exemple les travaux d'Espéret et Crété, 1995). Je mets à part les travaux qui se sont attachés à l'observation des temps de pause, en tant que celle-ci renseigne sur des styles différents de scription, car leur objet n'était pas de mesurer les modifications apportées par le recours au traitement de texte, mais d'identifier des modes de travail sur ordinateur (Doquet, 1995)

Enfin, en ce qui concerne le dernier critère, celui de la qualité des textes, critère combiné la plupart du temps aux deux observables mentionnés plus haut, là aussi les résultats furent décevants : certains rapports signalent que, non seulement il n'y a pas d'amélioration des textes lorsque la production s'effectue sur traitement de texte, mais qu'il y a même une détérioration de la qua-

9. Banque de données INRP-DAF. 
lité des textes, voire une détérioration des processus rédactionnels, ce que note Hayes:

Les études comparant la rédaction avec papier et crayon à la rédaction avec un traitement de texte ont fait apparaître les effets du medium sur les processus rédactionnels de planification et d'édition. Par exemple Gould et Grichowsky (1984) ont montré que les rédacteurs sont moins efficaces quand l'activité de production a été effectuée au moyen d'un traitement de texte plutôt que sur le papier [...] Haas (1987) a constaté que les étudiants planifiaient moins avant d'écrire lorsqu'ils utilisaient un traitement de texte plutôt qu'un papier et un crayon

(Hayes 1996 in Piolat \& Pélissier)

À la suite de ces résultats qui infirmaient les hypothèses optimistes concernant les effets du traitement de texte, la recherche s'est refocalisée sur le développement d'environnements informatiques plus complexes. Deux voies principales ont alors été explorées. La première portait sur l'étude des consignes destinées à accompagner la production sur traitement de texte (Roussey, 1989). La deuxième voie, qui a été explorée notamment par Crinon (Crinon et Pachet, 1995 ; Crinon et Legros, 2001) et par Mangenot (1995, 1996, $2000,2001)$, concernait la production de didacticiels intégrant des éléments du traitement de texte.

\subsection{Des discordances qui peuvent recevoir plusieurs types d'explications}

Sauf à admettre que les hypothèses mentionnées plus haut étaient mal fondées, il faut s'interroger sur les raisons des divergences entre ces hypothèses incitant à penser que le traitement de texte modifierait positivement les processus rédactionnels et les résultats enregistrés, d'autant plus que l'étude effectuée par Piolat rassure sur le sérieux méthodologique de la plupart des recherches (effectifs suffisants, présence de groupes témoins, tests...), en référence aux exigences attendues dans le cadre de la psychologie expérimentale.

Je vais donc dans un premier temps rappeler les causes mentionnées par Piolat pour expliquer ces résultats décevants, puis je proposerai quelques hypothèses complémentaires.

\subsubsection{Trois types de causes avancées pour expliquer ces discordances}

Les rapports de recherche examinés par Piolat mettent en avant trois grands types de causes:

\section{- Des causes d'origine matérielle}

Sont mis en cause les caractéristiques de l'affichage sur écran et les difficultés de manipulation dues au clavier. Les travaux de Woodruff et al. (1986, cité 
par Piolat, Isnard \& Della Valle, 1993) et ceux de Lutz (1987) attirent l'attention sur le rôle que joue l'écran qui, en quelque sorte, sélectionne des portions de texte soumises à l'attention du scripteur, et sur celui du curseur qui désigne des points d'intervention. Haas et Hayes (1986) ont également noté que la recherche en temps réel des informations dans le texte est fortement influencée par la taille de l'écran.

Je signalerai toutefois que les recherches évoquées plus haut se sont déroulées à une époque où la qualité des écrans était très inférieure à celle dont on dispose aujourd'hui, et que les interfaces graphiques n'offraient guère alors la possibilité du wysiwig, qui constitue actuellement une transition efficace entre les modes de traitement de l'information sur support traditionnel et sur écran.

En ce qui concerne les difficulté de manipulation, les recherches ont mis l'accent sur les problèmes de dactylographie que rencontraient les scripteurs, et particulièrement les scripteurs débutants. Ces difficultés ont entraîné des conséquences qui allaient à l'encontre des hypothèses évoquées plus haut, qui prévoyaient que les scripteurs seraient libérés de la partie graphique de la tâche d'écriture et qu'ils pourraient allouer plus de ressources cognitives aux opérations de haut niveau : le constat a été fait que les dactylographes inexperts consacrent au contraire une partie de leurs ressources cognitives au traitement des problèmes d'ordre dactylographique. Mais il s'agit là d'un point sur lequel je reviendrai, en l'abordant sous un autre angle.

De plus, le ralentissement imposé par les difficultés de frappe a des répercussions sur le rythme du traitement informationnel et provoque donc des dysfonctionnements processuels. De même qu'il est difficile pour un jeune enfant qui n'a pas automatisé les gestes graphiques de planifier son écrit et de le réviser en cours de scription parce que sa mémoire de travail est longtemps occupée par de courts segments textuels qu'il s'auto-dicte, de la même façon, le dactylographe débutant est accaparé par la lenteur de sa production. Ce constat valide les modèles de production qui se sont attachés à décrire la simultanéité partielle d'opérations de niveaux différents dans l'activité d'écriture.

\section{- Des causes d'origine humaine:}

Parmi les causes expliquant les résultats décevants, certaines sont imputées à l'inexpertise des rédacteurs participant aux expérimentations. Deux types d'inexpertise ont été pointées, celles qui concernent les aspects proprement rédactionnels, et celles qui portent sur la maîtrise des fonctionnalités du traitement de texte.

L'inexpertise rédactionnelle est indépendante du contexte imposé par le traitement de texte, mais les difficultés qu'elle génère et celles suscitées par les manipulations techniques ne font que se cumuler.

Les inexpertises du second type, qui concernent la maitrise technique de l'outil, affectent aussi bien les rédacteurs débutants que les rédacteurs aguerris: les conclusions de l'enquête de Joram citée par Piolat, Isnard et Della Valle 
(1991), réalisée en 1989 et portant sur des collégiens âgés de 13 ans ayant deux ans de pratique de l'ordinateur, vont dans le même sens que celles de l'enquête menée par Anis (1993) auprès des chercheurs : les deux enquêtes ont mis en évidence la sous-utilisation des facilitations offertes par le traitement de texte.

\section{- Des causes dues aux particularités de la tâche imposée}

Enfin ce dernier type de cause signalé par Piolat paraît peu dépendant de la machine : il s'agit des difficultés imputables aux contraintes d'écriture, comme celles concernant par exemple la nature de la consigne ou la longueur du texte attendu.

Crinon et Legros (2001) ont, dans leur revue de travaux sur la question, corroboré dans l'ensemble les résultats enregistrés par Piolat, mais leur constat est un peu plus nuancé. Cela tient au fait qu'ils se sont davantage intéressés à un autre facteur, celui de l'apprentissage, et qu'ils en montré le poids. Ils ont en effet pris en compte deux autres données du contexte, la temporalité et le contexte pédagogique, montrant ainsi que les expérimentations qui se sont attachées au seul medium ne parvenaient pas à en mettre en évidence les effets, alors que celles qui envisageaient le traitement de texte comme un outil dont l'utilisation devait s'apprendre avaient plus de chance d'obtenir des effets vérifiables.

\subsubsection{Des aspects de l'écriture non pris en compte}

Je vais maintenant revenir sur ces discordances entre les effets attendus et les effets avérés de l'écriture sur traitement de texte en essayant d'aborder la question sous un autre angle, qui sera celui du linguiste et du didacticien, et non pas celui du psycholinguiste, même s'il s'agit d'expériences réalisées dans le cadre de la psycholinguistique.

Je défendrai l'idée que ces discordances entre les effets attendus du traitement de texte sur la production d'écrit et les effets constatés sont dues non seulement aux causes évoquées plus haut, mais également à d'autres, incluant ou non les précédentes, que je vais rapidement évoquer. Mon propos n'est donc pas d'ajouter une liste de causes qui prolongerait celles que je viens d'énumérer, mais plutôt de la réorganiser, ou d'envisager dans une perspective différente les résultats de recherches mentionnées plus haut.

- Discordances entre résultats attendus et résultats constatés dues au fait que l'ordinateur ajoute une difficulté.

- L'affichage à l'écran : difficulté matérielle ou changement de fonctionnement sémiotique?

Revenons sur les problèmes liés au fait que l'affichage sur écran détermine la portion du texte à traiter et focalise l'attention du lecteur, l'empêchant en quelque sorte de prendre en compte le texte dans son intégralité. 
Il est incontestable que les conditions matérielles de lecture à l'écran influent sur l'activité rédactionnelle : les dimensions du texte soumis à relecture sont précalibrées, et les parties rédigées ou en cours de rédaction occultées ne sont accessibles qu'au prix d'une manipulation supplémentaire. Mais au-delà du coût mémoriel occasionné par le dispositif technique, il est intéressant de noter que l'ordinateur impose un véritable changement de système sémiotique en obligeant le scripteur à procéder sur du texte virtuel, comme le notait Anis (1995). En effet, le texte, tel qu'il est traité sur ordinateur, connait trois états de réalité qui exigent des traitements sensiblement différents de ceux requis par l'écriture sur papier:

- le texte déjà produit et non affiché est une entité linguistique dont la trace matérielle n'est accessible qu'au prix d'une manipulation technique, ce qui amène le scripteur à, en quelque sorte, doubler le stockage matériel par un stockage mémoriel plus exigeant que celui requis par l'écriture manuscrite et à travailler bien plus à partir de l'image mentale du texte produit qu'à partir des traces physiques du texte ;

- le texte affiché est une entité linguistique consultable, mais dont la mutabilité, l'instabilité est plus manifeste que dans le cas de l'écriture manuscrite ;

- le texte à venir est une virtualité paradoxale : d'une part ses contours sont encore plus imprécis que dans le cas de l'écriture manuscrite, car l'espace qui lui est offert est illimité ; d'autre part, il peut lui être assigné des caractéristiques plus contraignantes que celles qu'on imposerait à une écriture manuscrite, et en particulier sa longueur peut être déterminée au caractère près.

Ainsi, l'écriture sur traitement de texte contraint le scripteur non seulement à opérer un traitement mémoriel différent de celui requis par l'écriture sur papier, mais aussi à intégrer d'autres modes de fonctionnement sémiotiques, à partir d'une écriture largement dématérialisée. Ce n'est donc qu'au prix d'une longue habituation que le scripteur peut parvenir à procéder comme on le faisait à l'époque où le volumen obligeait le lecteur à conserver en mémoire du texte sinon absent du moins difficilement consultable (cf. Vanderdope, 1999). Notons que cela peut aussi être un choix : Kerouac écrivait, parait-il, non sur des feuilles mais sur des rouleaux de papier qu'il insérait dans sa machine à écrire, pour bénéficier de l'impression de rapidité que lui procurait le déroulement et le masquage en continu du texte au cours de sa progression.

On peut aussi pour compléter cette analyse, et l'envisager d'un point de vue didactique, considérer qu'il y a deux manières de traiter le problème, soit en termes de difficultés, soit en termes d'obstacles, auxquels on pourra attribuer une fonction positive dans l'apprentissage, en considérant que le fait que l'affichage soit limité amène le scripteur à développer de nouvelles compétences.

- Le clavier et la dimension graphique de l'écriture : des facteurs de complexité à réexaminer

Parmi les difficultés pointées par les expérimentations, la question de la maitrise de la dactylographie reste encore un thème de débat, avec, d'un côté, 
les partisans d'un apprentissage préalable de la dactylographie et, de l'autre, ceux qui en contestent l'utilité, soit parce qu'ils y ont eux-mêmes échappé, soit parce qu'ils redoutent les effets de la dissociation entre les apprentissages strictement techniques et les apprentissages scripturaux.

Je voudrais ici simplement attirer l'attention sur deux problèmes que même une bonne maîtrise technique du clavier ne peut juguler : l'inadaptation du clavier au geste graphique, et le problème de la désegmentation des ligatures.

Quatre caractéristiques du clavier alourdissent la tâche cognitive du scripteur:

- Les claviers d'ordinateurs sont organisés pour ralentir la frappe - ou la rendre plus compliquée, comme on veut - en proposant un ordre des lettres hérité des contraintes imposées à leurs ancêtres les machines à écrire mécaniques : cet ordre avait été établi de façon à ralentir la frappe pour éviter que les marteaux ne se percutassent les uns les autres. On pourrait donc dire que l'ordre des lettres fossilisé sur les claviers usuels de l'ordinateur est antiergonomique comme le prouve a contrario l'existence de claviers véritablement ergonomiques ${ }^{10}$, qu'il s'agisse de claviers physiques comme le maltron ou le mal irane, sur lesquels la disposition des lettres tient compte de la longueur des doigts, ou de claviers logiques comme le dvorak employé par les programmateurs (la frappe n'exige pas de commande biclave, les parenthèses sont à l'emplacement du $P$ et du $O$, c'est-à-dire au bout du majeur et de l'annulaire...).

- La non correspondance entre l'ordre des lettres mémorisé lors de l'apprentissage de l'alphabet et l'ordre des lettres tel qu'il figure sur le clavier (A, Z, E...) oblige le dactylographe débutant à passer d'un ordre mental à un autre, ce qui complexifie sa tâche.

- La forme générique des caractères figurant sur les claviers ne correspond pas à la forme des caractères qui s'inscriront sur l'écran (on doit frapper des touches du clavier portant les formes « $A$ », « $B$ ", « $E$ » etc. pour voir s'afficher les formes « $a$ ", “ b ", «e ") d'où l'obligation d'une opération de translittération proprement dite à effectuer qui s'ajoute à l'acte d'écriture, et qui, ainsi que j'ai pu le constater, n'est pas anodine pour de jeunes scripteurs.

- Enfin, comme le note Anis (1995) la frappe sur la frappe sur ordinateur fait de l'écriture une méta-écriture, en raison de son caractère analytique. Ainsi, contrairement à ce qui se passe lorsqu'on écrit à la main, vouloir taper un $A$ majuscule oblige à décomposer cette intention en l'opérationnalisant au moyen de deux gestes successifs, celui correspondant à la prise de décision " écrire en majuscule " (frappe sur la touche majuscule) et celui correspondant à la décision " graphier un $A$ " (frappe sur la touche A).

10. Les concepteurs de matériels informatiques qualifient d'ergonomiques les supports courbes de claviers. Mais il s'agit que du boîtier qui est plus ou moins ergonomique, non du clavier lui-même. 
Enfin, le caractère analytique de l'écriture sur traitement de texte produit deux effets à prendre en compte parce qu'ils influent sur la qualité rédactionnelle :

- L'écriture sur traitement de texte atomise les mots en parcelles disjointes et fait donc perdre de vue les unités plus importantes que sont les syntagmes et même les mots. L'observation de dactylographes débutants montre à l'évidence que la focalisation de l'attention sur de petites unités graphique accapare les scripteurs, même experts en rédaction.

- La déglobalisation de séquences graphiques automatisées perturbe l'écriture. En effet, je fais l'hypothèse qu'en dehors des segmentations imposées par le fonctionnement codique, il existe des ligatures mémorisées par chacun des scripteurs, et qui ne correspondent pas nécessairement à des assemblages morphologiques. II s'agit plutôt de formes graphiques propres au geste du scripteur. Leur dislocation imposée par la frappe lettre à lettre rompt un automatisme, d'où des interversions de lettres, en particulier lors de la frappe de mots dont l'usage est familier au scripteur. J'ai recueilli de nombreux témoignages de scripteurs qui déclarent faire des interversions récurrentes à chaque fois qu'ils dactylographient des mots dont ils ont automatisé la graphie, tel le cas de cet inspecteur amené à programmer la correction automatique du mot " professeur " sur son ordinateur, pour juguler sa tendance irrépressible à antéposer le u.

- Discordances entre résultats attendus et résultats constatés dues aux conceptions de l'écriture et du texte qui ont présidé aux expérimentations

Sur ce point je renverrai tout particulièrement aux analyses de Fabre (1990) qui a fait une critique sévère des conceptions de l'écriture présidant à certaines expérimentations, ainsi qu'aux mises en garde de Bereiter et Scardamalia qui, dès 1987 dénonçaient les illusions engendrées par une analogie abusive entre le fonctionnement des traitements de texte et les modélisations de l'activité d'écriture :

The computer memory dump (in which the contents of the computers's memory locations are written out seriatim) provides a convenient but completely wrong metaphor for the knowledge-telling process. We bring the matter up only because the metaphor is apparently very attractive to people who approach writing from an information processing standpoint. Human beings cannot list the contents of their memories - least of all young children, with their more limited memory-search procedure

Je complèterai leurs analyses par quelques remarques concernant plus précisément la manière dont ont pu être évalués les changements dans la production lors de certaines expérimentations sur les effets du traitement de texte. 
- La confusion entre la révision et les traces de révision

II s'agit là d'un point problématique soulevé par Roussey (1999) dans la synthèse qu'il a faite des travaux portant sur la révision. II signale en effet l'ambiguiité du terme, qui désigne, selon les auteurs, soit un ensemble d'opérations cognitivo-langagières réalisées sur un texte en cours de production, soit, par un raccourci hâtif, le résultat de cette activité (i-e. les transformations opérées sur ce texte).

Reprenant, sous un autre angle, des remarques faites par Brassart (1991) et par Bronckart (1985) qui mettaient en garde contre les conséquences possibles d'un parti-pris théorique hérité de Bloomfield et amenant à établir une corrélation directe entre les unités linguistiques de surface et les fonctionnements langagiers sous-jacents, Roussey met ainsi l'accent sur le glissement sémantique de " révision " opéré dans un certain nombre de travaux : ce terme, employé pour désigner les traces à partir desquelles l'analyste infère qu'il y a eu ou non telle activité mentale, permet de faire allégrement une incursion du côté des processus mentaux, la polysémie du terme "révision " autorisant ainsi à passer un peu rapidement de l'observation de transformations à des conclusions portant sur l'activité cognitive du scripteur. Pourtant, différentes recherches (voir par exemple, Bond, Hayes \& Flower, 1980) ont mis en évidence que des sujets pouvaient passer du temps sur un texte, en ayant pour consigne de le réviser, sans produire la moindre modification. Faut-il, pour autant, considérer que ces sujets n'ont pas réalisé la tâche qui leur était demandée ? Et l'observation de brouillons d'écrivain montre de nombreux exemples de cas où le mot choisi définitivement par l'auteur est celui-là même qui avait figuré dans une première version, avant d'être remplacé par un autre puis rétabli à sa place initiale. Je renverrai ici volontiers à un texte de Michel Butor ${ }^{11}$ publié dans ses Essais sur le roman disant qu'il commence sa révision à partir du moment où il se trouve en possession de schémas, c'est-à-dire avant d'être passé à la textualisation, puisque pour lui, dans son mode d'écriture, les différents types de macro-processus sont disjoints. On peut donc supposer que même lorsqu'il n'y a pas de traces graphiques, il doit arriver à des scripteurs de produire une formulation, d'envisager de la modifier, puis de décider de la conserver, privant ainsi l'observateur de données tangibles lui permettant d'apprécier l'activité rédactionnelle : les modifications opérées sur du texte, et les pauses ne sont donc que des indices, fragiles, et non des brèches ouvertes dans la boîte noire.

\section{- La question de la " qualité " du texte}

Cette question est un corollaire de la précédente. L'appréciation des effets du traitement de texte sur les processus rédactionnels a été faite soit en se fondant sur le nombre et la pertinence des " révisions" (c'est-à-dire, plus exactement, des phénomènes interprétés comme des traces de révision), soit en faisant appel à des jugements de qualité sur les textes produits (conformité aux

11. Intervention à Royaumont en 1959, repise dans BUTOR M. (1992 réed. 1997) Essais sur le roman. Gallimard. 
consignes, adaptation au destinataire...). Le plus souvent ces jugements de qualité ont été portés par des scripteurs experts, mandés pour comparer plusieurs états d'un texte ou plusieurs textes. C'est là une méthode qui offre un certain nombre de garanties, mais qui n'a guère plus de validité que n'en aurait un vote : les travaux de docimologie et ceux menés en didactique du français nous ont appris à nous méfier des appréciations sur la qualité des textes, soumises aux effets de halo, de stéréotypie ou d'autres facteurs viciant le jugement, et ce quelle que soit l'expertise de l'aréopage convoqué. Seule la détermination préalable de critères et d'indicateurs linguistiques très fins permet de décrire convenablement et de comparer des productions écrites.

\section{- La partition entre structure et surface}

Les analyses de résultats se fondent également sur une distinction entre correction de surface et correction de structure. II s'agit là d'une distinction qui ne recouvre en rien la distinction hjelmselvienne entre substance et expression, ou qui la caricaturerait de manière telle qu'elle serait indéfendable sur le plan linguistique. Lorsqu'on examine de plus près les grilles qui servaient à évaluer les transformations opérées lors de l'écriture, on a la surprise de voir souvent mis en œuvre des schémas très réducteurs, qui font l'économie de l'analyse des changements sémantiques. En particulier, les travaux de Witte et Faigley (1981) qui se fondaient sur la définition que Halliday et Hasan ont donné de la cohésion textuelle ont été figés en une sorte de grille mécaniste qui trahit leurs auteurs et leurs inspirateurs. Par exemple, on y apprend que les changements de topicalisation ou les variations énonciatives ne sont pas considérés comme affectant le sens du texte, ou du moins ils sont considérés comme des changements de surface, surtout si leur manifestation n'est repérable que dans le déplacement très court syntagme ou d'un changement de marque morphologique...

- Un postulat idéologique : la préexistence des « idées " sur leur actualisation

Je voudrais attirer l'attention ici sur un point particulièrement important qui concerne le postulat idéologique sur lequel se sont fondées certaines expérimentations. En effet, certains travaux reposent sur une conception sous-jacente du langage qui postule la préexistence des idées sur leur actualisation par le langage, comme si l'activité langagière n'était rien d'autre qu'une sorte d'encodage. Cette conception, qui sous-tend certaines expérimentations s'appuyant sur des modèles frustes de l'activité langagière, ne leur permet pas d'apprécier l'écriture en tant que travail conceptuel. Mais ce binarisme n'est pas le propre des recherches expérimentales : d'une part, certaines philosophies du langage ${ }^{12}$ s'en réclament, d'autre part, il est fortement inscrit dans la culture pédagogique, comme le montre l'analyse de Perrenoud Aebi (1997) qui a enquêté sur les représentations des enseignants sur l'écriture et son apprentissage.

12. Le discours sur le Style de Buffon en 1753 en est un bon exemple. 


\section{- La temporalité de l'écriture}

Sur le plan méthodologique, on note que dans les expérimentations la dimension temporelle n'est pas toujours pleinement prise en compte, alors qu'il est probable que les changements d'ordre processuel doivent s'inscrire dans la durée et il est donc normal qu'ils ne soient pas perceptibles après seulement quelques sessions d'écriture.

D'autres éléments importants ne sont pas pris en compte, mais c'est le propre d'une situation expérimentale que de faire en sorte que les variables soient contrôlées, et qu'en conséquence, la situation soit extrêmement contrainte. Toutefois dans le cas de l'écriture cela est problématique, dans la mesure où il y a presque antinomie entre d'une part, la dimension personnelle de l'écriture, sa nécessaire maturation, l'engagement qu'elle demande au sujet, sa dimension sociale, comme nous l'ont montré notamment les travaux de didactique, et, d'autre part, les contraintes méthodologiques de l'expérimentation.

\section{SUR QUELS POINTS CONVIENDRAIT-IL D'ENGAGER DE NOUVELLES RECHERCHES?}

Je voudrais maintenant signaler quelques thèmes sur lesquels il serait utile que des recherches s'engagent, car on ne dispose actuellement que d'hypothèses qu'il conviendra de vérifier ou d'infirmer. Je ne mentionnerai que les chantiers qui intéressent au premier chef la didactique.

Voici donc une petite liste de cinq suggestions pour inciter à des recherches futures.

\subsection{L'identification de types de scripteurs sur traitement de texte}

Comme on le sait, en raison des contraintes méthodologiques, dans bon nombre de recherches, les dimensions idiosyncrasiques de l'écriture ont été écrasées par les protocoles expérimentaux, alors qu'il s'agit d'une activité éminemment subjective, dans tous les sens du terme, ce qui fait que les travaux consacrés au traitement de texte se sont jusque là peu intéressés à la variété des scripteurs,

On a cependant des raisons de penser qu'il existe très probablement des styles de scripteurs sur traitement de texte, comme il en existe pour l'écriture manuscrite. II serait très précieux pour la didactique de l'écriture de disposer, soit de typologies, soit d'éléments de caractérisation adaptés, tenant compte, par exemple, de la manière dont le scripteur utilise à la fois les représentations du texte disponibles (affichage sur écran, tirage sur papier), et les traces mémorielles du texte enregistré ; dont il entame le processus d'écriture, par un plan, par la rédaction de l'incipit ou d'un segment central ; dont il traite les difficultés en les prenant en charge dès leur détection ou en les différant... Pourraient entrer dans ce champ d'étude également les aspects organisationnels de l'écri- 
ture (avant-textes manuscrits ou non, etc. la génétique fournissant les outils de description appropriés), ainsi que la gestion par le scripteur de certains problèmes particuliers tels les conséquences syntaxiques et sémantiques des déplacements de segments...

On dispose actuellement de trois typologies complémentaires qui devraient aider à caractériser des styles de brouillonneurs sur traitement de texte :

- la distinction ancienne de Bridwell-Bowles (1987) puis Johnson Brehe qui considère deux catégories, les beethoveniens et les mozartiens ;

- les distinctions établies dans le cadre de la génétique textuelle, qui ont d'abord reposé sur les deux grands modèles d'écriture proposés par Hay (1979), l'écriture à programme - comme celle de Zola qui élabore des plans minutieux et successifs avant d'écrire - et l'écriture à processus - comme celle de Proust qui brouillonne des pages jusqu'à trouver l'entrée du roman -, puis se sont complexifiées, certains généticiens allant même jusqu'à mettre en doute la possibilité d'une entreprise taxinomique (Boie et Ferrer 1993) ;

- la distinction établie par Doquet (1995) qui s'intéresse à la temporalité de l'écriture sur traitement de texte, ce qui l'a amenée à identifier deux grands profils, celui de l'écriture en deux temps, et celui de l'écriture sans rupture.

\subsection{L'émergence de nouveaux genres textuels}

Il semble qu'il y ait des genres textuels qui se prêtent mieux que d'autres à l'écriture sur traitement de texte : le travail sur traitement de texte uniformise les signes graphiques, évacuant ainsi la trace personnelle graphique du geste du scripteur, et contribuant ainsi à mettre à distance le texte. Cet aspect anonyme du graphisme est un avantage si l'écrit à produire est impersonnel, mais ce peut être une gêne si le texte projeté a quelque chose d'intime qui engage la personne du scripteur. Mais il peut se créer une proximité entre le scripteur et son ordinateur qui l'autorise à en faire un confident, comme l'a montré le recueil de témoignages collectés par Lejeune auprès de personnes tenant leur journal intime sur ordinateur.

II conviendrait donc que s'engagent des recherches s'intéressant au traitement de la distance du scripteur avec son écrit lorsqu'il est produit sur traitement de texte, la génétique textuelle et la linguistique de l'énonciation fournissant les outils d'analyse nécessaire pour étudier cet aspect de l'écriture. Une deuxième piste de recherche concernerait la description des formes textuelles émergentes. Je forme en effet l'hypothèse que le recours au traitement de texte devrait favoriser le développement de structures narratives qui soit existaient auparavant mais avaient un statut expérimental, soit émergeront à la faveur des possibilités techniques offertes par l'outil. Je pense en particulier aux récits entrecroisés, aux récits à structure fractales, disjointes, dans lesquels le traitement des incidentes reçoit un statut particulier. Ainsi, par exemple, l'écriture de Rouaud, celle d'Eco bien sûr, celle de Bello ou de Lurie entre autres, utilisent de façon manifeste des types de constructions favorisées par le traitement 
de texte (insertions, auto-citation, fragmentation et recomposition...), et font bouger ainsi les genres fictionnels disponibles.

\subsection{L'observation des conséquences des changements sémiotiques ou grapho-sémiotiques imposés par le traitement de texte}

L'espace offert à la scription par le traitement de texte est à la fois infini et labile. II est infini non seulement en longueur mais aussi en profondeur : le texte peut se creuser à tout endroit pour laisser s'insérer de nouveaux fragments qui dilatent non seulement l'énoncé, mais aussi l'espace de scription. Cet espace est aussi labile, car il peut se réduire sous l'effet des manipulations de suppression, qui ne laissent de traces que dans la mémoire du scripteur, mais dont le texte affiché ne garde nul témoignage. On peut donc dire que les scripteurs travaillent sur des espaces graphiques virtuels dont la délimitation est assurée non par des contraintes matérielles, mais par le texte, qui, en quelque sorte génère son propre espace.

Il devrait être intéressant que s'engagent des recherches portant d'une part sur le rôle organisateur, structurant, de la disposition spatiale, et, d'autre part, sur la dynamique autorisée ou suscitée par la possibilité de modifier la disposition en cours de production. Ces recherches pourraient également porter sur les aspects grapho-sémiotiques de l'écriture en s'attachant à observer l'effet sur la production textuelle et sur les textes eux-mêmes de la possibilité d'user des techniques graphiques au cours de la scription : la possibilité de recourir à des formes de titrage, à des outils de mise en forme au cours même de la session d'écriture ne peut pas être sans influence sur la nature de la production. Devraient être également étudiés l'effet des limitations imposées par le traitement de texte (impossibilité d'utiliser certains modes de signalement, de reprendre une feuille déjà en partie écrite en la plaçant la tête en bas comme le faisait Michel Leiris dans ses brouillons manuscrits...) et l'effet des contraintes exercées par les logiciels qui tendent parfois à prendre des décisions typographiques à la place des scripteurs (création de listes, uniformisation formelle des paragraphes etc.), et imposent des modèles typo-rédactionnels (modèles de courriers pré-construits etc.).

\subsection{L'évolution des modes de coopération et de dialogue dans l'écriture}

Des recherches pourraient s'attacher à étudier l'évolution des pratiques d'écriture et en particulier d'écriture à deux, comme cela se produit par exemple dans le cadre de collaborations scientifiques. L'écriture en collaboration s'opère selon des modalités variées qui ont été analysées au sein du GRIC, notamment par de Gaulmyn grâce à un dispositif expérimental permettant d'observer des interactions entre des scripteurs spatialement distants, mais bénéficiant d'une liaison directe assurée par le biais de connexions entre leurs ordinateurs. La voie se trouve ainsi ouverte à des travaux portant sur les spécificités de la coopération rédactionnelle sur traitement de texte, qui pourraient s'engager sur des 
thèmes complémentaires. Ainsi, il serait utile de disposer de typologies permettant de décrire les modes de d'organisation du travail d'écriture sur traitement de texte, en prenant éventuellement comme point de référence ce que l'on sait de l'écriture manuscrite qui nous offre des modèles très variés, depuis Erckmann et Chatrian se spécialisant l'un dans la recherche de la trame narrative, l'autre dans la mise en texte, Stevenson réclamant de Fanny Osborne l'invention orale d'aventures qu'il thésaurisait pour les narrer plus tard, ou à l'inverse, Dumas fournissant à Maquet un plan à expanser, et prenant en charge éventuellement les réductions imposées par son éditeur, ou encore Boileau et Narcejac travaillant sur le mode de l'échange et de la successivité... Outre la répartition des fonctions rédactionnelles dans les groupes d'écriture, il serait intéressant de voir la manière dont les formes de coopération sont influencées par ce nouvel outil d'écriture qui autorise, grâce à l'insertion et au déplacement de blocs, la restructuration hiérarchique du contenu informationnel des textes. Ces situations de coopération peuvent également être étudiées dans une perspective dialogique sous l'angle des types et modalités d'insertion du discours d'autrui dans son propre discours, menant à observer également comment, sur traitement de texte, un scripteur dialogue avec lui-même.

\subsection{Le traitement mémoriel du texte et les effets de coupé-collé}

Le traitement de texte de texte offre la merveilleuse et trompeuse facilité d'autoriser sans limite les déplacements de blocs, permettant ainsi la mise à l'épreuve d'effets de sens liés au positionnement mouvant des segments textuels. Mais cette souplesse n'est pas sans danger : le texte peut souffrir des perturbations dont il fait l'objet, et le scripteur est donc contraint à une vigilance sans relâche, d'autant plus que son activité s'exerce non pas sur un texte tangible, mais sur une épaisseur infinie de virtualités. Il est donc amené à construire des architectures mouvantes, sans cesse révisables. En outre, alors que dans l'écriture manuscrite tous les vestiges issus du travail matériel de production sont consultables, dans l'écriture sur ordinateur, la plus grande partie des ratures ou des modifications disparait du domaine du consultable et ne demeure qu'à l'état de traces mémorielles.

Les conséquences de cette situation pourraient faire l'objet d'études descriptives. Ainsi, il serait intéressant d'une part de rechercher les indices qui renseignent comme des cicatrices malheureuses sur les déplacements inaboutis, et d'observer d'autre part comment s'opère le traitement de la cohérence quand il ne s'appuie plus sur la linéarisation, la successivité du texte.

Le traitement de texte, qui a suscité beaucoup de travaux lors de sa mise sur le marché, puis lors de son introduction à l'école, est aujourd'hui quelque peu délaissé par la recherche, comme s'il était devenu en quelque sorte transparent. Cela est dû sans doute à la déception qu'il a causée en ne révélant pas être l'outil miracle qui résoudrait tous les problèmes d'écriture. Maintenant que la déception a été digérée, il est temps de s'y intéresser de plus près, en l'abordant avec les outils d'analyse de la linguistique. C'est là une nécessité didactique : l'école ne pas continuer à enseigner l'écriture en ignorant les spécificités 
de cet instrument d'écriture appelé à être l'un de ceux qu'utiliseront le plus les élèves une fois qu'ils auront quitté l'école.

\section{BIBLIOGRAPHIE}

ANIS J. (1993) : “Des scripteurs professionnels face au traitement de texte. Représentations et pratiques des enseignants-chercheurs ». Les Cahiers Pédagogiques 311.

ANIS J. (1995) : “ Le traitement de texte : écriture ou méta-écriture ». INRP, Repères 11.

ANIS J. (1998) : Texte et ordinateur. L'écriture réinventée. Bruxelles, De Boeck.

BARRE DE MINIAC C. (1999) : Copie et modèle : usages, transmission, appropriation de l'écrit. INRP.

BAYARD J, NICOLET M. et al. (1998) : L'informatique intégrée à l'enseignement du français. Enquête auprès des enseignants vaudois recourant à l'ordinateur pour des activités de français. Lausanne. CVRP

BEAUGRANDE (de) R. (1982) : “ Les contraintes générales qui affectent les processus de compréhension du langage ". In Bulletin de psychologie XXXV.

BEAUGRANDE (de) R. (1984) : “ Text Production : Toward a science of Composition ". Norwood, NJ Ablex in Scardamalia M.\&, Bereiter C.,1986, Research on written composition in Wittrock M.C., Amarel M. Handbook of Research on Teaching, New-York, Macmillan.

BEREITER C. et SCARDAMALIA M. (1987) : The Psychology of Written Composition. Hillsdale, Lawrence Erlbaum.

BISAILLON J. (1991) : “ Les stratégies de révision comme objet d'enseignement ». Namur, Enjeux 22.

BOND S.J., HAYES J.R. et FLOWER L.S. (1980) : Translating the law into common language : A protocol study. Document Design Project I, Pittsburgh, PA : Carnegie Mellon University.

BOIE B. et FERRER D. (1993) : “Les commencements du commencement ». In Genèses du roman contemporain - Incipit et entrée en littérature. Paris, CNRS-ITEM.

BORGOGNON M. (1998) : Écrire avec un traitement de texte. Une revue de la littérature. Lausanne, CVRP.

BRIDWELL L.S., JOHNSON P. et BREHE S. (1987) : “ Computers and composing : Case studies of experienced writers. " In A. Matsuhashi (Ed.), Writing in real time: Modelling production processes. New York : Longman.

BRONCKART J.P. (dir.) (1985) : Le fonctionnement des discours. Delachaux et Niestlé

COHEN M. (dir.) (1963) : L'écriture et la psychologie des peuples. Actes du XXII Colloque du Centre International de synthèse du 3 au 11 mai 1960. Paris, Armand Colin.

COIRIER P., GAONAC'H D. et PASSERAULT J.-M. (1996) : Psycholinguistique textuelle. Approche cognitive de la compréhension et de la production des textes. Paris, Armand Colin. 
CRINON J. et LEGROS D. (2001) : « De l'ordinateur outil d'écriture à l'ordinateur outil ». INRP, Repères 22.

CRINON J. et PACHET S. (1995) : “L'aide à l'écriture ». INRP, Repères 11.

DAIUTE C. (1981) : "Psycholinguistic Foundations of the Writing Process ". In Research of the Teaching of English. Vol 15, $n^{\circ} 1$, février 1981.

DAVID J. et PLANE S. (Eds.) (1996) : L'apprentissage de l'écriture de l'école au collège. Paris, PUF.

DOQUET C. (1995) : " Le temps d'écrire : stratégies d'écriture et chronologie des événements dans des processus d'écriture d'élèves de CM2 ". INRP, Repères 11.

ESPÉRET E. (1991) : " Improving Writing Skills : Which Approaches and What Target Skills ? " European Journal of Psychology of Education. Vol. VI, 2.

ESPÉRET E. et CRÉTÉ M.-F. (1995) : “ Processus cognitifs mis en jeu dans la production écrite : sont-ils modifiés par le traitement de texte ? "INRP, Repères 11.

FABRE C. (1990) : Les brouillons d'écoliers ou l'entrée dans l'écriture. Grenoble, Céditel.

FABRE-COLS C. (2002) : Réécrire à l'école et au collège. de l'analyse des brouillons à l'écriture accompagnée. ESF.

FAYOL M. et GOMBERT J.E. (1987) : “ Le retour de l'auteur sur son texte. Bilan provisoire des recherches psycholinguistiques". INRP, Repères 73.

GELB. I.J. (1963 ; trad. 1973) : Pour une théorie de l'écriture. Paris, Flammarion.

GODINET H. (2000) : “ Hypermédias et parcours labyrinthiques ». Le français Aujourd'hui 129.

GOODY J (1977, trad. 1979) : La raison graphique. La domestication de la pensée sauvage. Paris, Éditions de Minuit.

GROUPE EVA (1996) : De l'évaluation à la réécriture. Hachette-INRP.

HAAS C. et HAYES J.R. (1986) : « What did I just say ? Reading Problems in writing with the Machine ". Research of the Teaching of English, 20.

HAY L. (dir.) (1979) : Essais de Critique Génétique. Paris, Flammarion.

HAYES J.R. (1996) : « Un nouveau cadre pour intégrer cognition et affect dans la rédaction ". In PIOLAT $A$. et PÉLISSIER A. (dir.) (1998) : La rédaction de textes. Approche cognitive. Lausanne, Delachaux et Niestlé.

HAYES J. R. et FLOWER L.S. (1980) : “The Dynamics of Composing : Making Plans and Juggling Constraints ". In Gregg L.W, Steinberg Cognitive Processes in Writing. Hillsdales, Laurence Erlbaum.

De LANDSHEERE G. (1974) : Évaluation continue et examens : Précis de docimologie. Paris, Nathan.

LAUFER R. et SCAVETTA D. (1992) : Texte, hypertexte, hypermédia. Paris, PUF.

LEJEUNE P. (2000) : “ Cher écran... » Journal personnel, ordinateur, Internet. Paris, Seuil.

LEROI-GOURHAN A. (1964) : Le geste et la parole. Technique et langage. Paris, Albin Michel.

LÉVY P. (1990a) : Les technologies de l'intelligence. l'avenir de la pensée à l'ère informatique. Paris, La découverte. 
LÉVY P. (1990b) : "L'hypertexte comme technologie intellectuelle et métaphore ". In Informatique et différences individuelles (collectif). Presses Universitaires de Lyon. 59-68.

LUTZ J.A. (1987) : " A study of Professional and Experienced Writers Revising at the Computer and with Pen and Paper ». Research in the Teaching of English, 21.

LUSIGNAN G. et FORTIER G. (1990) : Révision de textes en fonction d'un changement d'audience au secondaire. Université du Québec à Montréal. Rapport de recherche.

MANGENOT F. (1995): Aides logicielles pour apprentis scripteurs en langue maternelle et en langues étrangères. Thèse de doctorat en sciences du langage sous la direction de $J$. ANIS, Université de Paris $X$.

MANGENOT F. (1996) : Les aides logicielles à l'écriture. Paris, CNDP.

MANGENOT F. (2000) : “Aide à l'écriture ou environnements d'écriture ? "In ANIS J. et MARTY N. : Lecture-écriture et nouvelles technologies. Paris, CNDP.

MANGENOT F. (2001) : L'ordinateur, instrument de manipulation(s) linguistiques(s). INRP, Repères 22.

MATSUHASHI A. (1982) : " Exploration in the Real-Time Production of written Discourse ". In Nystrand M. (Ed.) What Writers know. The language Process and Structure of Written Discourse. New York, Academic Press.

MORO C., SCHNEUWLY B. et BROSSARD M. (1997) : Outil et signes. Perspectives actuelles de la théorie de Vygotski. Berne, Peter Lang.

OLSON D.R. (1994 trad. 1998) : L'univers de l'écrit. Comment la culture écrite donne forme à la pensée. Paris, Retz.

PAPERT S. (1981) : Jaillissement de l'esprit. Ordinateurs et apprentissage. Paris, Flammarion.

PERRENOUD Aebi C. (1997) : Enseigner l'écriture. Paroles d'enseignants. Université de Genève. Cahier de la section des sciences de l'éducation $n^{\circ} 84$.

PIOLAT A. (1991a) : « Écrit-on mieux avec un ordinateur? » In M. Fayol (éd.) : La production de textes écrits. Dijon, CRDP.

PIOLAT A. (1991b) : "Effects of Word processing on text revision ", Language and Education. Vol 5, $\mathrm{n}^{\circ} 4$.

PIOLAT A., ISNARD N. et DELLA VALLE V. (1993) : “ Traitement de texte et stratégies rédactionnelles ", Le travail humain, t. 56, n 1/1993.

PIOLAT A. et PÉLISSIER A. (dir.) (1998) : La rédaction de textes. Approche cognitive. Lausanne, Delachaux et Niestlé.

PIOLAT A. et ROUSSEY J.-Y. (1991) : “Écrit-on mieux avec un ordinateur? »Le Journal des psychologues, 86.

PLANE S. (1994) : Didactique et pratiques d'écriture - Écrire au collège. Nathan.

PLANE S. (1996) : “ Écriture, réécriture et traitement de texte ". In DAVID J. et PLANE S. (éds.) : L'apprentissage de l'écriture de l'école au collège. Paris, PUF.

PLANE S. (2001) : Permanence(s) et mouvements des traces langagières. Investigations sur la production langagière, ses traces et ses outils. Dossier 
d'Habilitation à diriger des Recherches. Sous la direction de jacques Anis, Université Paris $X$.

PLANE S. et SCHNEUWLY B. (2001) : “ Regard sur les outils de l'enseignements du français - Un premier repérage ". INRP, Repères 22.

POUDER M.C. (1995) : “ L'écriture sur ordinateur et ses représentations chez des élèves de CM2 en atelier informatique ". INRP, Repères 11.

PRÉFONTAINE C. (1987) : Effets de l'utilisation du traitement de texte sur la pratique de l'écriture. Rapport de recherche. Cégep de Valleyfield.

RABARDEL P. (1997) : “ Activités avec instruments et dynamique cognitive du sujet ". In Moro C., Schneuwly B. et Brossard M., Outil et signes. Perspectives actuelles de la théorie de Vygotski. Berne, Peter Lang.

ROSAT M.C., DOLZ J. et SCHNEUWLY B. (1991) : “ Et pourtant... ils révisent ! Effet de deux séquences didactiques sur la réécriture de textes ». INRP, Repères 4.

ROUET J.F (1997) : “ Le lecteur face à l'hypertexte ". In CRINON J. et GAUTELLIER C. : Apprendre avec le multimédia. Où en est-on. Paris, Retz.

ROUET J.F. (2000) : « Hypermédias et individualisation des apprentissages : quels prérequis cognitifs ? ", Le français Aujourd'hui 129.

ROUSSEY J.-Y. (1999) : Le contrôle de la rédaction de textes. Perspective cognitive. Dossier d'habilitation à diriger des recherches, sous la direction d'A. Piolat. Université d'Aix.

SCHNEUWLY B. et PFEIFFER V. (2001) : « Les outils de l'enseignant - Un essai didactique ". INRP, Repères 22.

SNYDER I. (1993a) : “Writing with Word Processors : A research Overview » Educational Research 35, 1.

SNYDER 1. (1993b) : "The impact of Computers on Student Writing : A comparative Study of the Effects of Pens and Word Processors on Writing Context, Process and Product ". Australian Journal of Education 37.

TURCO G, MAS M. et PLANE S. (1994) : “Construire des compétences en révision- réécriture au cycle 3 de l'école primaire ". INRP, Repères 10.

VAN GALEN G.P. (1990) : "Phonological and motoric Demands in handwriting : Evidence for discrete Transmission of Information ". Acta Psychologica, 74, présenté dans FAYOL M. (1997) : Des idées au texte. Psychologie cognitive de la production verbale, orale et écrite. Paris, PUF.

WITTE S.P. et FAIGLEY (1981) : “ Coherence, Cohesion and Writing Quality ". College Composition and Communication 32.

WOODRUFF E., LINDSAY P., BRYSON M. et JORAM E. (1986) : " Some cognitive Effects of Words Processors on Enriched and Average 8th Grade Writers ". Communication présentée à l'Annual Meeting of the American Educational Research Association, San Francisco (citée in Piolat, Isnard \& Della Valle). 